\title{
Adaption of Big Data Technology for ITG Trust Framework: A Coherent Framework
}

\author{
Resad Setyadi \\ \{resad setyadi@gmail.com\} \\ Bung Karno University
}

\begin{abstract}
Utilization of Big Data technology in the industrial era 4.0 is basically used to get huge profits in data processing and utilization. In Big Data technology, processing and utilizing large volumes of data, fast changing data, varied data, and data complexity are indispensable for the effectiveness and efficiency of service strategies, preparation of IT infrastructure, and needs of corporate governance or ITG. On the other hand, ITG trust aims to provide timely, reliable, meaningful and adequate data services. Apparently, this goal is the same as the goal of Big Data technology services offered to support ITG. We propose an ITG trust framework influenced by Big Data technology. The ITG trust framework presents criteria different from existing criteria at the data quality level. It focuses on timely, reliable, meaningful, and sufficient data services, focusing on what data trust attributes should be achieved based on the data trust attributes of Big Data services. In addition to the quality level of Big Data, the personal information protection strategy and the data disclosure/accountability strategy are also needed to achieve goals and to prevent problems. This paper performed case analysis based on the ITG trust Framework on the National Education Service in High School Institution. Big Data services in the public sectorare especially on education field hope to improve the quality of student education's in that country. ITG trust and its trust framework are the essential components for the realization of Big Data service.
\end{abstract}

Keywords: big data, ITG, trust framework, case analysis.

\section{Introduction}

Educational institutions in the industrial era 4.0 must know more and equip their capabilities with information technology (IT). IT is a bridge to lead to Big Data technology. Educational institutions can process and utilize data with large amounts of data, variations in data, different levels of data understanding, and fast flow of data transfers so that millions of bytes of data are created every second from various sources, such as internet, video and smart data. Educational institutions that are able to process and utilize information technology through big data technology can take advantage of the benefits and importance of data quality as part of one of the decision makers. Through ITG, educational institutions try to adopt, integrate and adapt of Big Data technology for the purposes of processing and utilizing the data.

ITG has been described as an effective and efficient resource and IT process governance to facilitate the achievement of the target goals [1], but scholars mention that there are still some items influenced by the trust domain [2]. In particular, the authors identified four conditions that need to be improved around the ITG domain of trust; first, ITG has a high 
level of risk because it is less successful; second, the definition of trust in ITG does not yet have a clear definition of trust; third, ITG dimension measurements are different because several authors have different usage concepts; fourth, the dimensions of ITG has different ITG identification methods.

\section{Literature review}

\subsection{Information Technology Governance (ITG)}

IT governance provides a structure for institutional education in preparing and ensuring IT investments to support the institution's plans, objectives and business implementation. Conceptually, IT governance offers and equips the structure with the aim of aligning business strategies with IT strategies. The implementation of IT governance is carried out by following a formal framework procedure, so that educational institutions can produce measurable results towards achieving the -TI business strategy. The formal program of aligning IT-business strategies also considers the interests of stakeholders, as well as the needs of staff and the processes they follow. In short, IT governance is an integral part of the governance of educational institutions or organizations as a whole and formal IT governance programs must also be able to train the consistency of the focus of any institution or organization that is related to actions to comply with regulations related to financial accountability and technology. As it turns out, to conduct a comprehensive IT governance program requires a lot of time and effort. Starting from a very small entity factor that may only practice essential IT governance methods, up to the goals of larger and more organized institutions or organizations must become a complete IT governance program.

Modern institutions have Deep understanding of IT and persisting in dealing with problems. Furthermore, the institution to use information as a way of solving the problems at hand IT and innovation are topics that are recognized by the public and private sectors around the world as drivers for improving public services and improving business performance [3]. The role of individuals in aligning business-IT and ITG is important to make symmetry and interrelation between business and IT to produce quality IT business values.

ITG is the main focus of current educational institutions to produce an understanding of IT that comes from IT investments by increasing IT mastery capabilities. Daily institutional habits indirectly affect the performance of the ITG in the direction of success or not and this is also related to the use of IT economically in its investment costs. The current position of stakeholders in educational institutions for IT is that they must be able to function as regulators of modern IT and provide equal portions in strategic and operational roles. ITG has a simple concept of institutional expertise in its efforts to create symmetrical and strategic IT benefits and business progress through IT [4].

\subsection{Trust}

Trust is as a psychological measurement to make up a decision that whether it should interact or take the risk that might turn out to be misplaced and harm. On the other word, trust is considered as a relationship between trustor and trustee [2]. There are connected between human, social sciences and computer science, social status and physical properties and objective factors such as competence and reputation. Trust features is judging whether a trustee (a person, a device or a service) is trustable or not, then the trustor "thinks" like human 
by taking its knowledge, recommendations from trustor's relations; and trustee's reputation into account. Assessing trust is imitated in trust model by modulating reputation, recommendation, and knowledge as basic of trust governance.

\subsection{Information Technology Governance Trust (ITG Trust)}

In particular, the authors identified four conditions that need to be improved around the ITG domain of trust; first, ITG has a high level of risk because it is less successful; second, the definition of trust in ITG does not yet have a clear definition of trust; third, ITG dimension measurements are different because they have different usage concepts; fourth, the dimensions of ITG that have different ITG identification methods.

Based on these identified conditions, the idea emerged that how ITG development would be better to increase consumer confidence in this case is the parents of students and students towards educational institutions. The coherent ITG trust framework will be developed based on four points of consideration: first, guidance on the definition of trust; second, integrating the three dimensions of trustworthiness, privacy, and trustworthy stakeholder attributes in a coherent manner; third, adapting the relationship between the definitions of attributes of trust and determination of trust in ITG; fourth, adopting ITG perceptions from high school institution stakeholders.

A trust framework needs to be created to support the ITG trust model as a tool for evaluating ITG performance in high school institutions. The literature review described is derived from the previous ITG concept and is a development of the proposed framework. The last part of this paper is shown by concluding the suggestions for further study.

\subsubsection{The Five Condition That Need to be Improve Around ITG Trust}

First, the non-existent of ITG in optimized stages and in initial stages rate only $6 \%$ all were at the good managed [5]. The next researchers said that the solution to address IT sourcing issues from the ITG is underutilized [6]. Therefore, the researcher identifies this condition that need to be improve, by explicitly tie IT sourcing to ITG. The influence of ITG is reliable to the knowledge and positively increases high expectation for education business institution. However, when the ITG experience is not reliable, then the effect is perceived as negative.

Second, scholars mentioned that education institution failure in ITG because they are not flexible in aligning their ITG to business-IT in daily implementation [7]. This effect can influence the performance in to positively innovation so the stakeholder efficiently try to use another way to maintain the ITG in every their institution. Innovation implementation is major to influence institutional performance. IT enhances to effort and detects the possibility of developing the use of skills and technology. Efforts to detect the possibility of developing the use of skills and technology will be connected to ability of IT determines the quality of results from IT governance which are all related to strategic decisions taken.

Third, several researchers used the trust attribute to describe the trust [8]. Without trust attributes, then ITG can not to be ensured by user's has effect positively secure communications and trustworthy interactions. Accordingly, ITG trust becomes a crucial challenge to privacy services and reliable security. Quality of information (QOI), quality of system (QSY) and quality of service (QSV) as three variable of usability in system use to support trust variable in ITG trust model that will be support the high content validity of ITG trust [9]. 
Fourth, to propose trust as a relevant input in a ITG trust model development [9]. Trust indicator is developed by adopt trust attribute to ITG trust model. Dynamic trust attributes adoption in the ITG trust model to deliver trust when ITG implemented.

Fifth, to facilitate to user that still believe because of they are not fear of their data and their privacy still secure [10]. The author argues still needs a trust framework for understanding ITG trust model. Moreover, it will help ITG stakeholder in dealing with the complexity of governance senior high school institution environment.

\subsection{Big Data}

Big Data is a cost-effective, high-speed and high-volume asset that has the ability to store variety information by processing data with all the innovations needed to improve insight and decision making [11]. A new data management strategy is needed to cope with the growth of data on the internet, social media, and open data. Big Data technology is a response to the need for the management strategy. In the field of education, the use of Big Data needs to be integrated into the master plan for aspects of the development of balanced and long-term education corresponding to information governance and Big Data analytics capabilities on innovation [12]. The simple way of working with big data is as follows:

1. Collect and filter internet data that is structured and unstructured.

2. Processing data to convert unstructured data into structured and eliminate dark data (data that is not needed).

3. Useful information is generated from processing data.

4. The formulation and evaluation of policies is made from the analysis of information obtained

The relationship between Data governance, ITG, and ITA / EA is shown in Figure 1. Figure 1 explains the interrelationships of information data governance, IT governance techniques, and the interrelated forms of IT architects implemented in the diagram. The attributes of the trust framework adapted from big data technology include security, stakeholders, and privacy. In the governance of information data, the system is expected to be able to provide trust and protection in the trust of security, privacy, and stakeholder trust. Therefore, the authors adapt the attributes of security, privacy and stakeholders as attributes that form understanding related to trust. Each attribute turned out to be influenced by factors that play an important role in influencing the attribute of trust in a conceptual and applied role on the framework of trust

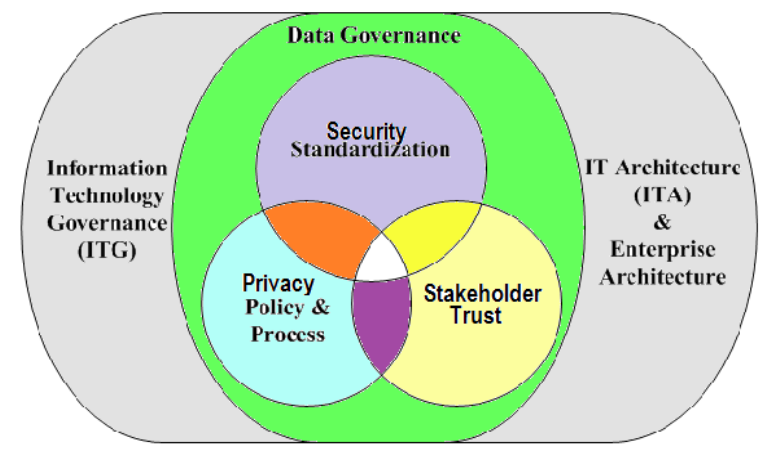

Figure 1. Relationship between Data Governance, ITG, and ITA/EA 
The eight benefits of using Big Data technology in education institution are:

1. to collect, filter and perform functions data mapping

2. to predict analysis in real time and dynamically

3. to become the source of data aspirations

4. as an educational data center that record;
a. academic side
b. psychology
c. social
d. lifestyle trends of students

5. to help the Ministry of Education and Culture (MOEC) carry out timely and useful policy formulation.

6. to guide parent of students how to do the right family approach to students.

7. to design programs for students.

8. to guide teachers to make learning method

\section{Proposed Trust Framework}

Based on the early pages and reviewing the previous literatures, authors adapted framework attribute by implemented several factors of attribute Big Data technology to trust framework as presented by Fig 2. The steps adoption, integration and adaption from Big Data technology factors to trust framework, i.e. Firstly, authors define that ITG trust is a governance which is implemented by the best attribute of secure level, privacy level and stakeholder trust worthies level [9]. The aim is to produce a good ITG result from trust attributes that fulfill of ITG the functional requirements. Based on this definition, authors identify three trust attribute that support ITG trust model, i.e. security, privacy and stakeholder trust worthies. These attributes will be guidance for formulating the trust framework that influent by Big Data technology factors. Secondly, the trust framework is made by integrated from 6 attribute level coherently to encourage the high content on ITG trust measurement. The attribute compositions of trust level attributes are; reputation, continuity, good suggestion/recommendation, personal qualities /credentials, association/delegation, and belief. The attribute compositions of privacy level are respondent privacy, user privacy, owner privacy, ethical, policy, and laws. The attribute compositions of security level are available, able to receive/non repudiation, integrity, confidential, authentic, and approval/ authorization.

The use of trust framework is to describe the ITG performance when it is used to assess the ITG in school institution. Thirdly, authors adopt, integrate and adapt the Big Data technology factors to build the trust framework in business -- IT alignment and IT governance [4]. The aimed is to know how the factors of Big Data influenced the trust level of stakeholder in business -- IT alignment. The eighteen attributes are bring downed based on the three formulated trust framework and influenced by Big Data technology factors of ITG attribute dimensions as represented by Table 1.

Adaptation of Big data technology to the trust framework in technology governance trust can be seen from three elements of trust framework in technology governance trust. Three attributes derived from big data technology have six factors that influence each attribute. 
Table 1. Trust Framework Attribute

\begin{tabular}{lll}
\hline Attribute & Factors & Reference \\
\hline Stakeholder & Reputation & {$[13]$} \\
& Continuity & {$[14]$} \\
& Good Suggestion & {$[15]$} \\
& Personal & {$[16]$} \\
& Association & {$[17]$} \\
& Belief & {$[18]$} \\
Privacy & Respondent & {$[19]$} \\
& User privacy & {$[20]$} \\
& Owner privacy & {$[21]$} \\
& Ethical & {$[22]$} \\
& Policy & {$[23]$} \\
& Law & {$[24]$} \\
Security & Available & {$[25]$} \\
& Able to receive & {$[26]$} \\
& Integrity & {$[27]$} \\
& Confidential & {$[28]$} \\
& Authentic & {$[29]$} \\
& Approaval & {$[30]$} \\
\hline
\end{tabular}

Factors that influence each of the forming attributes of the trust framework appear to be interrelated between the factors of the attributes. The factor of attribute stakeholders has a relation to the factors of the privacy attribute, and the factor of the stakeholder attribute has a relation to the security attribute factors. Because of this connection, the trust framework is created by adopting factors, integrating factor attributes, and finally adapting them into a trust framework as shown in Figure 2.

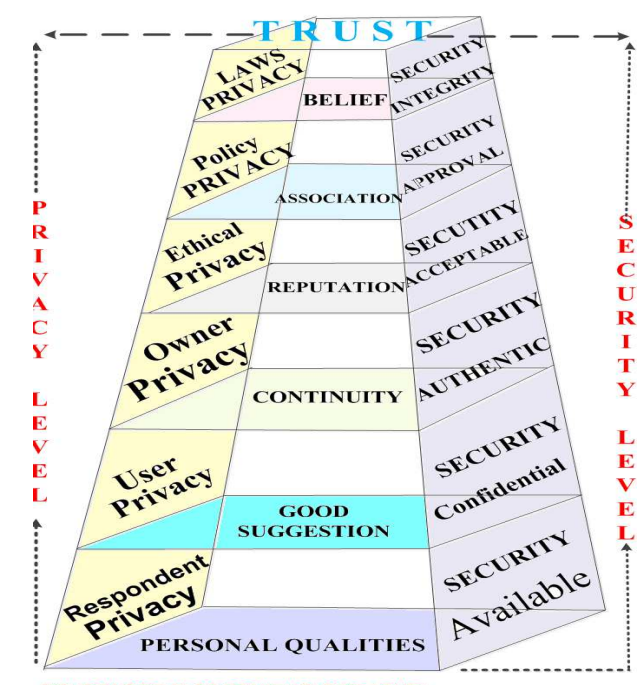

TRUSTWORTHY OESTAKEHOLDEER LEVEL

Figure 2. ITG trust framework 


\section{ITG Trust Model}

Adaption of Big Data technology in trust framework to support ITG trust model shown in Figure 3. Figure 3 shows the model of ITG trust with its 9 variables and 23 relational hypotheses and one of the varable is trust variable.

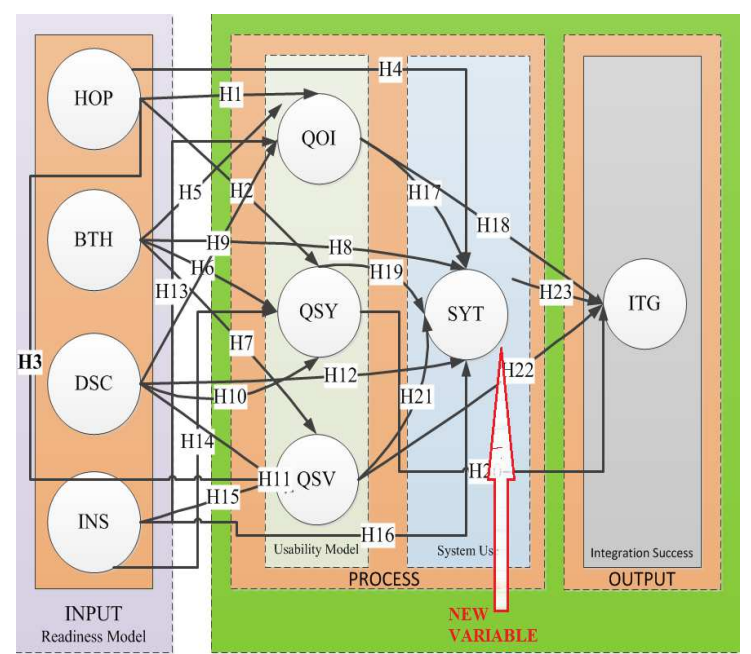

Figure 3. ITG Trust Model [9]

The development model resulted from the integrating of the readiness model (parasuraman) and usability models (Nielsen). Variable that comes from the readiness model, i.e. Hopefulness (HOP), Breakthrough [BTH], Discomfort [DSC], and Insecurity [INS]. On the other hand, several of variable that comes from the usability model, i.e. contains Quality of Information [QOI], Quality of System [QSY], Quality of Service [QSV]. The results of the integration will be added by the system Trust [SYT]) variable. Existing trust variables have three attributes with six factors each on attributes derived from adaptation of big data technology. The last result from the integration of readiness model variable, usability model variable and trust variable is ITG trust variable. The relational hypothesis emerges which is then developed to meet influential relationships between models.

\section{Result And Discussion}

This research produced a composition of a trust framework that was formed from the adaptation of big data technology. The trust framework in the next step is used as the forming variable of the IT governance trust model. The three trust attributes formulating the trust framework that influent by Big Data technology. The trust framework attribute is made by integrated from 6 factors level coherently to encourage the high content on IT governance measurement. 
In increasing trust level in IT governance, there needs to be a change in the user's perspective through various kinds of education and training that refers to all kinds of changes in systems that are influenced by technology. Based on the existence of a trust framework, it is expected that the criteria for evaluating trust in the use of the system can be identified and used as a reference for assessing the extent to which a person trust in IT governance in a school institution or company. Thus, the role of technology in helping the system work properly can be achieved. 


\section{References}

[1] S. J. Andriole, "IT Governance in the Trenches," IEEE Journals \& Magazines, vol. 18, pp. 57 - 62, Mar.-Apr 2016.

[2] G. M. Lee, Trust in ICT. Geneva Switzerland International Telecommunication Union, 2017.

[3] S. Borja, K. Kim, and H. Yoon, "IT Governance Effectiveness and Its Influence on Innovation Product and Process " in Portland International Conference on Management of Engineering and Technology (PICMET), Honolulu, HI, USA, 2018, pp. 1-8.

[4] H.-T. Wagner and J. Meshtaf, "Individual IT Roles in Business -- IT Alignment and IT Governance " in 49th Hawaii International Conference on System Sciences (HICSS), Koloa, HI, USA, 2016, pp. 4920 - 4929.

[5] W. Yaokumah, "Information Technology Governance Barriers, Drivers, IT/Business Alignment, and Maturity in Ghanaian Universities," International Journal of Information Systems and Social Change, vol. 7, p. 22, 2015.

[6] A. Sesay and R. Ramirez, "Theorizing the IT Governance Role in IT Sourching Research," presented at the Twenty-second Americas Conference on Information Systems, San Diego, 2016.

[7] S. Héroux and A. Fortin, "The moderating role of IT-business alignment in the relationship between IT governance, IT competence, and innovation," Information Systems Management vol. 35 , p. 26, 2016.

[8] N. B. Truong, T.-W. Um, B. Zhou, and G. M. Lee, "From Personal Experience to Global Reputation for Trust Evaluation in the Social Internet of Things," presented at the IEEE Global Communications Conference, Sing apore, 2017.

[9] R. Setyadi, A. a. Subiyakto, and A. B. A. Rahman, "Assessing the Information Technology Governance Trust Using Readiness and Usability Models: A Model Development Study," in international conference on ICT for rural development, denpasar bali, 2018, pp. 131 - 136.

[10] D. De Smet and N. Mayer, "Integration of IT governance and security risk management: A systematic literature review," in International Conference on Information Society (iSociety), Dublin, Ireland, 2016, pp. 143 - 148.

[11] H. Y. Kim and J.-S. Cho, "Data Governance Framework for Big Data Implementation with a Case of Korea " in IEEE International Congress on Big Data (BigData Congress), Honolulu, HI, USA, 2017, pp. 384 - 391.

[12] P. Mikalef, G. Lekakos, and J. Krogstie, "Complementarities Between Information Governance and Big Data Analytics Capabilities on Innovation," presented at the TwentySixth European Conference on Information Systems (ECIS2018), Portsmouth,UK, 2018.

[13] F. Alam and A. Paul, "A computational model for trust and reputation relationship in social network," in 2016 International Conference on Recent Trends in Information Technology (ICRTIT), Chennai, India, 2016, p. 6.

[14] P. Zhang, K. Zhao, and R. L. Kumar, "Impact of IT Governance and IT Capability on Firm Performance," Infromation Systems Management, vol. 33, p. 17, 2016.

[15] H. Wu, P. Ren, and Z. Xu. (2019, Hesitant Fuzzy Linguistic Consensus Model Based on Trust-Recommendation Mechanism for Hospital Expert Consultation. IEEE Transactions on Fuzzy Systems ( Early Access ).

[16] T. Nakanishi and T. Kanatani, "An Efficient Blacklistable Anonymous Credential System with Reputation Using Pairing-Based Accumulator," in 2018 17th IEEE International 
Conference On Trust, Security And Privacy In Computing And Communications/ 12th IEEE International Conference On Big Data Science And Engineering (TrustCom/BigDataSE), New York, NY, USA, 2018.

[17] M. Schanzenbach, C. Banse, and J. Schütte, "Practical Decentralized Attribute-Based Delegation Using Secure Name Systems," in 2018 17th IEEE International Conference On Trust, Security And Privacy In Computing And Communications/ 12th IEEE International Conference On Big Data Science And Engineering (TrustCom/BigDataSE), New York, NY, USA, 2018, pp. 244 - 251.

[18] L. Ding, O. Savas, G.-S. Ahn, and H. Deng, "Securing Cognitive Radio Networks with Distributed Trust Management against Belief Manipulation Attacks," in 2015 IEEE Globecom Workshops (GC Wkshps), 2015, pp. 1-6.

[19] N. Kato, Y. Murakami, T. Endo, and K. Nawa, "Study on privacy setting acceptance of the drivers for the data utilization on the car," in 2016 14th Annual Conference on Privacy, Security and Trust (PST), Auckland, New Zealand, 2016.

[20] S. Zaman, K. F. Khawaja, and S. Waqar, "Impact of service Quality Perceived Risk, Perceived Privacy and Security and Perceived Trust on technology acceptance model: An empirical study based on online banking sector of Pakistan," 2013, pp. 89 - 94.

[21] A. Kusyanti and N. D. Prastanti, "The role of privacy, security and trust in user acceptance of smartphone user in Indonesia," in International Conference on Information and Communication Technology (ICoIC7), 2017, pp. 1- 6.

[22] G. Kobayashi, M. E. Quilici-Gonzalez, M. C. Broens, and J. A. Quilici-Gonzalez, "The Ethical Impact of the Internet of Things in Social Relationships: Technological mediation and mutual trust," IEEE Consumer Electronics Magazine, vol. 5, pp. 85- 89, 2016.

[23] R. Vanickis, P. Jacob, S. Dehghanzadeh, and B. Lee, "Access Control Policy Enforcement for Zero-Trust-Networking," in 2018 29th Irish Signals and Systems Conference (ISSC), Belfast, UK, 2018.

[24] C. Gupta, "The Market's Law of Privacy: Case Studies in Privacy and SecurityAdoption," IEEE Security \& Privacy, vol. 15, pp. 78 - 83, 2017.

[25] H. Bin, S. Wong, C. Mannweiler, M. Dohler, and H. D. Schotten, "Security Trust Zone in $5 \mathrm{G}$ networks," in 24th International Conference on Telecommunications (ICT), Limassol, Cyprus, 2017.

[26] J. Li, "Factors Affecting Customer Satisfaction and Customer Loyalty towards Belle Footwear Company in Lanzhou City , Gansu Province of the People's Republic of China," Journal of Business and Management, vol. 14, pp. 41-48, 2013.

[27] G. Thangaraju, X. A. K. Rani, and J. Umarani, "Multi Level Multi Attribute Relational Trust Measure Based IntegrityManagement in Data Warehouse," in 2017 World Congress on Computing and Communication Technologies (WCCCT), Tiruchirappalli, India, 2017, p. 4.

[28] J. Y. Ryu, J. Lee, and T. Q. S. Quek, "Confidential Cooperative Communication With Trust Degree of Potential Eavesdroppers," IEEE Journals \& Magazines vol. 15, pp. 3823 3836, 2016.

[29] S. Sathyanandani and D. Sreedharan, "An E-commerce feedback review mining for a trusted seller's profile by classifying fake and authentic feedback comments," in International Conference on Circuit ,Power and Computing Technologies (ICCPCT), Kollam, India, 2017 pp. $1-6$.

[30] Y.-h. Wang, X. Cheng, and J.-y. Ye, "Research of trust authorization model in P2P network," 2012, p. 4. 